\title{
Mathematical Oncology: How Are the Mathematical and Physical Sciences Contributing to the War on Breast Cancer?
}

\author{
Arnaud H. Chauviere • Haralampos Hatzikirou • \\ John S. Lowengrub • Hermann B. Frieboes • \\ Alastair M. Thompson • Vittorio Cristini
}

Published online: 22 July 2010

(C) The Author(s) 2010. This article is published with open access at Springerlink.com

\begin{abstract}
Mathematical modeling has recently been added as a tool in the fight against cancer. The field of mathematical oncology has received great attention and increased enormously, but over-optimistic estimations about its ability have created unrealistic expectations. We present a critical appraisal of the current state of mathematical models of cancer. Although the field is still expanding and useful clinical applications may occur in the future, managing over-expectation requires the proposal of alter-
\end{abstract}

Arnaud H. Chauviere and Haralampos Hatzikirou contributed equally to this work

Dr. Cristini is an Honorary professor of Mathematics in the University of Dundee, Scotland

A. H. Chauviere $\cdot$ H. Hatzikirou $\cdot$ H. B. Frieboes $\cdot$ V. Cristini $(\triangle)$

School of Biomedical Informatics,

The University of Texas Health Science Center,

7000 Fannin Street,

Houston, TX 77030, USA

e-mail: Vittorio.Cristini@uth.tmc.edu

A. H. Chauviere

e-mail: Arnaud.H.Chauviere@uth.tmc.edu

H. Hatzikirou

e-mail: Haralampos.Hatzikirou@uth.tmc.edu

H. B. Frieboes

e-mail: Hermann.B.Frieboes@uth.tmc.edu

\section{J. S. Lowengrub}

Department of Mathematics,

The University of California at Irvine,

Irvine, CA 92697, USA

e-mail: lowengrb@math.uci.edu

\section{J. S. Lowengrub}

Department of Biomedical Engineering,

The University of California at Irvine,

Irvine, CA 92697, USA native directions for mathematical modeling. Here, we propose two main avenues for this modeling: 1) the identification of the elementary biophysical laws of cancer development, and 2) the development of a multiscale mathematical theory as the framework for models predictive of tumor growth. Finally, we suggest how these new directions could contribute to addressing the current challenges of understanding breast cancer growth and metastasis.
A. M. Thompson

Department of Surgical Oncology, M.D. Anderson Cancer Center, 1400 Holcombe Boulevard,

Houston 77030, USA

V. Cristini

Department of Systems Biology,

The University of Texas M. D. Anderson Cancer Center, Houston, TX, USA

\footnotetext{
V. Cristini

Department of Biomedical Engineering,

The University of Texas,

Austin, TX, USA
} 
Keywords Breast cancer $\cdot$ Mathematics $\cdot$ Physics . Oncology $\cdot$ Modeling $\cdot$ Multiscale

\section{Introduction}

The hallmarks of cancer include a disordered balance of cell proliferation and cell death resulting in tumor growth, direct tissue invasion, and metastasis. The initiation and sustenance of carcinogenesis requires multiple steps [1], suggesting not all breast cancers are the same [2]. Also, the concepts of cancer stem cells [3], invasion incorporating tumor-stroma interactions [4], and metastasis (including the self-seeding hypothesis $[5,6]$ ) are conceptually challenging. However, there remain significant gaps in our understanding of breast cancer and how best to manage the disease [7].

In addition to these more generalizable molecular and biological concepts of malignancy, breast cancer presents specific clinical problems of in situ disease (ductal carcinoma in situ [DCIS], lobular carcinoma in situ), distinct types of invasive cancer (including several different histologic types, transcriptome subtypes, or typing based on immunohistochemistry of estrogen receptor [ER], progesterone receptor $[\mathrm{PR}]$, and human epidermal growth factor receptor type 2 [HER2]), and metastatic disease (again this can be subclassified by site of metastasis to loco-regional skin/tissues/node, visceral, or bone). The balance of expectations of surgery, radiotherapy, and drug therapy improving survival from breast cancer against the clinical reality of a rising global (mostly female) breast cancer incidence, with breast cancer becoming a chronic but incurable disease for some, leaves room for significant improvements in our understanding of the biology and hence therapy of breast cancer.

The limitations of current strategies for managing breast cancer have moved beyond selecting the extent of locoregional surgery and radiotherapy to the need to improve the targeting of drug therapy. There remains uncertainty in identifying those patients who will actually benefit from targeted therapies and those for whom therapy will be ineffective or unnecessary. Despite many years of experience with ER and PR for endocrine treatments, and more recently HER 2 or other targeted therapies, such biomarkers still only define about $40 \%$ of patients who will be cured as a consequence of the therapy, and there are few promising markers of response to chemotherapy. Whether more recent genomic technologies have an impact on clinical practice remains to be seen [2].

At present, a large number of patients develop DCIS, invasive, and/or metastatic breast cancer for which there are some effective therapies, although $50 \%$ of women ultimately die from breast cancer. Despite significant clinical and research resources generating large amounts of data, breast cancer requires a refocusing of scientific endeavor; the mathematical and physical sciences could provide the much needed advance.

There is a substantial literature that addresses subcellular function in normal and transformed cells, but less that examines the bigger picture of the tissue heterogeneity and complexity that influences nutrient diffusion, drug diffusion, and cell migration. There is a pressing need to extend intracellular considerations to the physical meta-scale, including tissue architecture, and to up-scale this to the tumor, organ, and whole body, as well as the reverse process to account for the impact of tissue-scale processes on smaller scales. On an individual patient basis, this may translate into understanding the mechanisms and time-scale of cancer growth, and consequently how much tissue to resect (and where from); where to target radiotherapy and how local tissue characteristics (eg, composition) affect the efficacy of radiotherapy; how drugs, antibodies, or small molecules are transported through tissues into neoplastic cells; and the relationships between biological processes and therapeutic response.

\section{Use of Modeling Approaches}

Mathematical modeling provides a rigorous framework for understanding disease evolution and for testing biological hypotheses. By translating biological complexity and translating biological components of cancer development into mathematical terms, the modeling process describes cancer-related phenomena as a complex set of interactions with the emerging outcome predicted by mathematical analysis that defines the field of mathematical oncology [8]. This field is characterized by two main ideas: 1) that mathematics can be applied to improve biomedical knowledge of the disease, and 2) that biology proposes new mathematical challenges, which generate enhanced mathematical tools.

In regard to the first idea, two main approaches have been developed. Computational oncology uses mathematical techniques to extract information from large datasets (such as transcriptome, proteome, or imaging data) where extensive computational resources are utilized either by means of statistical and bioinformatics methodologies or for the study and quantitative prediction of tumor behavior by means of data-driven models $[9,10]$. Physical oncology views tumors as complex systems that result from biophysical interactions and processes. This leads to mechanism-driven models that aim at the identification and analysis of (bio)physical laws to quantify and predict cancer progression. 
Experimentation with a model is performed by changing the parameters of the system and studying the differences in outcomes of computer simulations, as the complexity of the models prohibits any mathematical analysis in all but the most simplified settings. Physical modeling attempts to provide a simplified description of reality to develop a better understanding of the various phenomena involved in cancer development. Both approaches are important to advance cancer research.

A boom in mathematical studies of cancer development occurred during the past decade $[11,12,13 \cdot]$. In this paper, we identify key publications in mathematical/ physical oncology (principally involving solid tumors as in breast cancer) and present a critical appraisal of the current state of the field. We have identified eight key topics for which we discuss the impact of modeling by means of selected publications that exhibit inspiration, originality, and completeness of approach and that, for some topics, constitute most of the few valuable studies (Table 1).

\section{Understanding Hypoxia-Induced Phenomena}

Hypoxia is a key driving force of tumor progression: tumorinduced angiogenesis, necrosis, invasion, or anaerobic metabolism are potential responses by tumor cells to hypoxic conditions. Gatenby and Glawinski pioneered the use of mathematical methods to study the influence of hypoxia on tumor evolution. In particular, they focused on the emergence of the anaerobic response by tumor cells to a low oxygen supply and the evolutionary advantage that such an adaptation may confer. By using a model of glycolysis associated with tumor development, it was shown how tumor growth is promoted through microenvironmental acidification [8]. This acid-mediated invasion was proposed as a simple mechanism linking altered glucose metabolism with the ability of tumor cells to form invasive cancers [14]. Such invasive tumors show an unstable morphology driven by heterogeneities of the environment due to non-uniform distribution of oxygen, captured in more recent modeling studies by Frieboes et al. [15].

Table 1 Key topics and selected publications for understanding the mathematical modeling of cancer

\begin{tabular}{|c|c|c|c|}
\hline Topic & Major finding & Selected publications & Clinical importance \\
\hline $\begin{array}{l}\text { Hypoxia-induced } \\
\text { phenomena }\end{array}$ & $\begin{array}{l}\text { Heterogeneous environment (eg, non-uniform } \\
\text { distribution of oxygen) and acid-mediated invasion } \\
\text { results in highly variable and complex tumor } \\
\text { behavior reproducing many clinical observations }\end{array}$ & $\begin{array}{l}\text { Gatenby and Gawlinksi [8] (2003); } \\
\text { Frieboes et al. [15] (2010) }\end{array}$ & $\begin{array}{l}\text { Radiotherapy, } \\
\text { chemotherapy }\end{array}$ \\
\hline Intra-tumoral transport & $\begin{array}{l}\text { Hypoxic regions, providing a source of angiogenic } \\
\text { factors, play a crucial role in the interaction between } \\
\text { tumor growth and the developing neovasculature }\end{array}$ & $\begin{array}{l}\text { Zheng et al. [18] (2005); } \\
\text { Cristini et al. [19] (2005); } \\
\text { Welter et al. [20] (2008) }\end{array}$ & $\begin{array}{l}\text { Chemotherapy, } \\
\text { radiotherapy }\end{array}$ \\
\hline Drug delivery & $\begin{array}{l}\text { Quantification of the diffusion barrier as an } \\
\text { explanation of poor response to chemotherapy }\end{array}$ & $\begin{array}{l}\text { Frieboes et al. [22] (2009); } \\
\text { Sinek et al. [21] (2009) }\end{array}$ & Chemotherapy \\
\hline Tumor size & Predicting tumor growth and tumor size & $\begin{array}{l}\text { Macklin et al. [25] (2010); } \\
\text { Szeto et al. [24] (2009) }\end{array}$ & $\begin{array}{r}\text { Imaging, } \\
\text { surgery }\end{array}$ \\
\hline $\begin{array}{l}\text { Mechanisms of tumor } \\
\text { progression }\end{array}$ & $\begin{array}{l}\text { Invasive cancers use multiple adaptive strategies } \\
\text { to overcome specific microenvironmental growth } \\
\text { constraints }\end{array}$ & $\begin{array}{l}\text { Gatenby and Gillies [29•] (2008); } \\
\text { Hatzikirou et al. [27] (2010) }\end{array}$ & Surgery \\
\hline $\begin{array}{l}\text { Interface tumor-host } \\
\text { tissue }\end{array}$ & $\begin{array}{l}\text { Understanding and implications of tumor growth } \\
\text { morphology }\end{array}$ & $\begin{array}{l}\text { Bru et al. [30] (2003); } \\
\text { Cristini et al. [31] (2003); } \\
\text { Bearer et al. [32] (2009) }\end{array}$ & $\begin{array}{l}\text { Chemotherapy, } \\
\text { radiotherapy, } \\
\text { surgery }\end{array}$ \\
\hline Cancer stem cells & $\begin{array}{l}\text { Implications of cancer stem cells on spatio-temporal } \\
\text { tumoral architecture and morphology }\end{array}$ & $\begin{array}{l}\text { Enderling et al. [34] (2009); } \\
\text { van Leeuwen et al. [33] (2007); } \\
\text { Galle et al. [35] (2009) }\end{array}$ & $\begin{array}{l}\text { Chemotherapy, } \\
\text { radiotherapy }\end{array}$ \\
\hline Multiscale modeling & $\begin{array}{l}\text { Hybrid multiscale modeling: the next generation } \\
\text { of tumor models }\end{array}$ & $\begin{array}{l}\text { Lowengrub et al. [13•] (2010); } \\
\text { Kim et al. [37] (2007); } \\
\text { Ramis-Conde et al. [38] (2008) }\end{array}$ & $\begin{array}{l}\text { Imaging, } \\
\text { surgery, } \\
\text { chemotherapy, } \\
\text { radiotherapy }\end{array}$ \\
\hline
\end{tabular}




\section{Prediction of Intra-Tumoral Transport}

Although oxygen gradients within the tumor bulk define hypoxic regions of critical importance for tumor development, more generally substrate transport within tumors critically affects tumor growth characteristics. The effect of nutrient supply by the vasculature of the tumor has been modeled by Byrne and Chaplain [16] based on one of the pioneering mathematical formulations generated to account for nutrient diffusion and consumption [17]. This original formulation [17] remains the basis of most current models. Importantly, Zheng et al. [18] advanced the first model coupling tumor growth with angiogenesis, allowing for the identification of hypoxic regions within a tumor. Similar formulations have been the basis of more refined and sophisticated studies of spatially heterogeneous cell proliferation and migration (eg, where microenvironmental substrate gradients may drive tumor morphology [19]) and have made possible the improvement of modeling drug delivery because of a better understanding of the effects of vascularization on tumor development [20].

\section{Drug Delivery and Impact on Tumor Growth}

Diffusion gradients of both drug and microenvironmental substrates induce physiologic resistance, diminishing the efficacy of drug therapy. Of particular importance is the "diffusion barrier effect," where diffusion gradients combined with highly packed cells increase drug resistance, which can result in a poor response to chemotherapy from a combination of diminished drug delivery and lack of nutrients required for cell proliferation (and drug activity). In addition, the poorly functioning tumor-induced vasculature can also prevent drugs from reaching the tumor. The studies of Sinek et al. [21] and Frieboes et al. [22] investigate in detail the modification of drug and substrate gradients within tumors and predict drug penetration correlated with drug efficacy (Fig. 1).

\section{Prediction of Tumor Size}

Resection of primary breast cancer remains the most effective therapy. Breast conservation raises the issue of how to identify the tumor margin, as margins are difficult to detect either by eye or by imaging techniques, reflecting the lack of encapsulation and the low density of tumor cells migrating into the breast stroma. Involved or close tumor margins on surgical resection correlate with tumor recurrence and poor clinical outcomes. A similar problem exists for other cancers (eg, glioma) where margin detection is also challenging. An ambitious goal of mathematical models has been the prediction of
Fig. 1 Validation of hypothesized functional relationships in a computational model of breast cancer drug response quantifying the important effect of physiologic resistance introduced by diffusion gradients of cell substrates and drug in threedimensional tumor tissue. The graphs show cell viabilities as a fraction of control versus doxorubicin (Dox) concentrations in A, drug-sensitive (MCF-7 WT) and $\mathbf{B}$, drug-resistant (MCF-40F) cells (glucose concentration $=$ $2.0 \mathrm{~g} / \mathrm{L}$ and time $=96 \mathrm{~h}$ of drug exposure). The in vitro monolayer without diffusion gradients is reported along with threedimensional in vitro tumor spheroids with diffusion gradients. Predictions made by the model are based on hypothesizing the resistance introduced by the gradients onto the monolayer data. (Adapted from Frieboes et al. [22]; with permission)
A

Cell viability (Drug-sensitive)

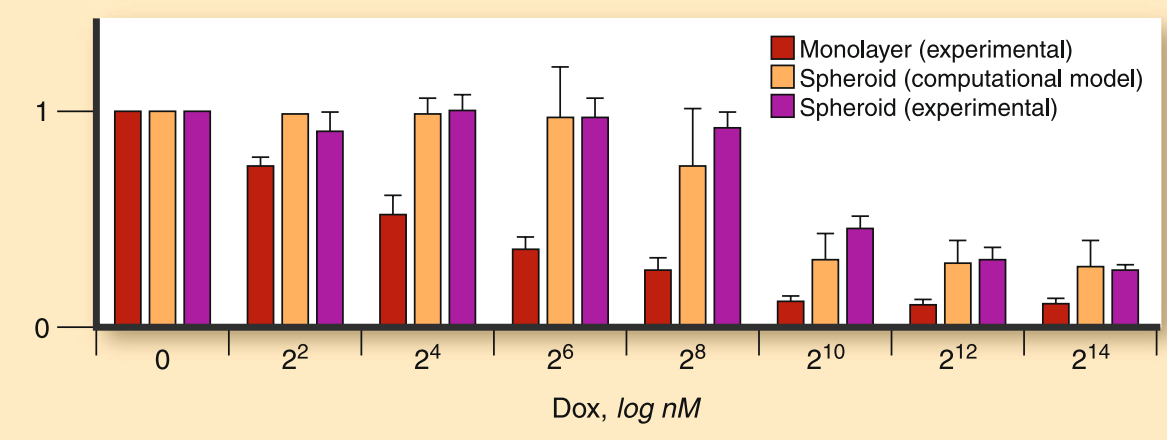

B

Cell viability (Drug-resistant)

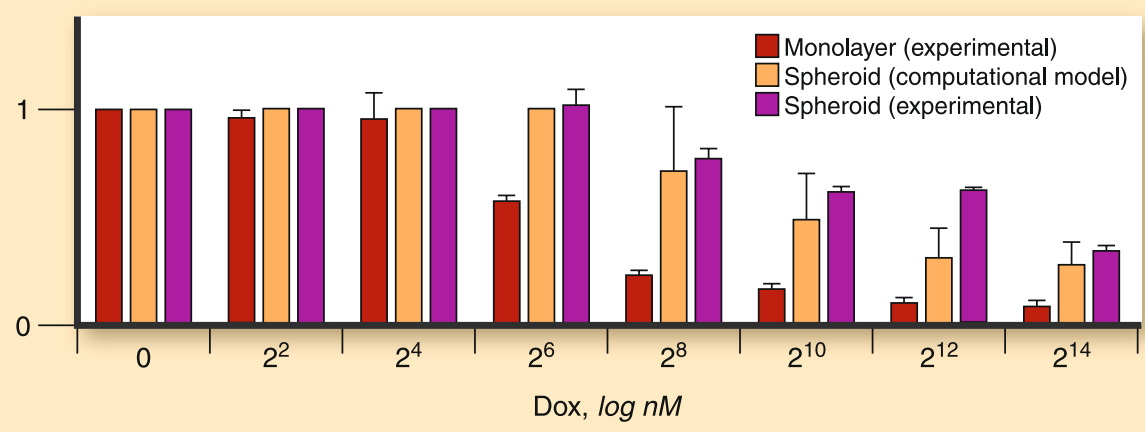


this "invisible" margin. Swanson et al. [23] developed a model of glioma growth by taking into account the preference of glioma cell migration along fiber tracks. By combining preoperative and postoperative imaging data with this tumor growth model, Szeto et al. [24] reported an accurate prediction of patient survival, fitting the model parameters for proliferation and migration, which could not entirely correlate with realistic values. An agent-based model was recently developed that considered in vivo cell-level data to predict clinical evaluation of breast tumor size without adjusting the model parameters [25]. This novel approach is one of the few trying to address the issue of size prediction, which remains unresolved.

Key Mechanisms of Tumor Growth and Evolution of Malignancy

The evolutionary transformation of healthy cells into cancer cells includes genetic mutations and epigenetic mechanisms involving gene up-regulation or down-regulation according to microenvironmental selective pressure [26]. The biological literature relies heavily on the importance of accumulation of mutations as the driving force toward malignancy. However, random genetic mutations may not explain recurrence, and the synergy of tumor substrate alterations with a specific cell mechanism (the migration/proliferation dichotomy) may indeed be responsible for recurrence, as proposed for glioma [27]. Although the influence of the microenvironment on tumor growth has been considered by several authors (Anderson et al. [28], Zheng et al. [18], Cristini et al. [19]), one of the most exciting works of how tumor microenvironment induces the emergence of "fit" phenotypes has been conducted by Gatenby and Gillies [29•]. Their work provides a theoretical framework where tumor adaptive strategies to circumvent microenvironmental growth constraints may result from genotype and phenotype heterogeneity.

\section{Interface of Tumor-Host Phenomena}

There is a consensus that most tumor cell activity is located at the interface between tumor and host tissue. High proliferation rates are observed close to the tumor margin, and invasive tumor cells escape from the margins of the tumor bulk. Therefore, a modeling effort has focused on this tumor-host tissue interface (Fig. 2). A mathematical characterization of the interface in terms of scaling exponents from studying various boundary microstructures in vivo and in vitro has been derived by Bru et al. [30]. These exponents provide information about the dynamics of the tumor interface, with the key result that all tumor interfaces may exhibit the same dynamics. Further elucidation is provided by a physical model that predicted the "fingering" morphology of invasive tumors into the surrounding tissues as a mechanical instability associated with cell-to-cell adhesion modeled by a surface tension [31]. One of the follow-up developments from the same

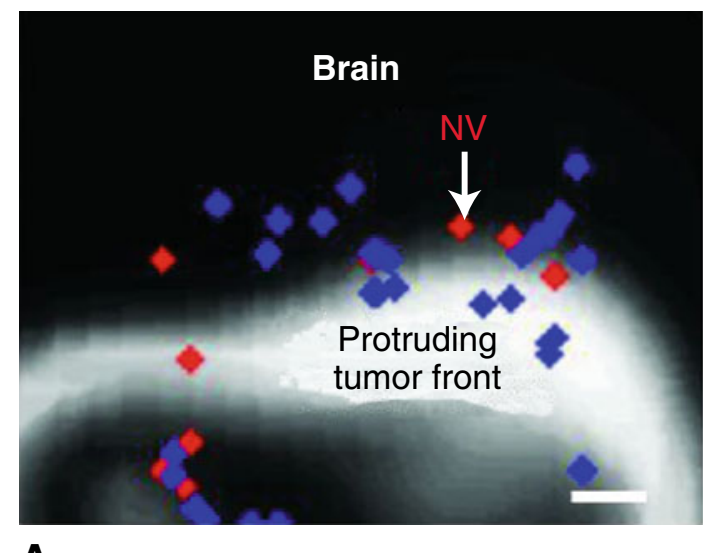

A

Fig. 2 Multiscale modeling has been considered in more detail for glioma (rather than breast cancer), where models predict that tumor invasiveness and morphology is strongly influenced by diffusion gradients of cell substrates. A, Detail of computer-simulated glioma histology showing protruding tumor front moving up toward extratumoral conducting neovessels (NV), supporting the hypothesis that diffusion gradients maintained by the neovasculature drive collective tumor cell infiltration in addition to determining the tumor structure. Aged vessels inside the tumor have thicker walls and thus are assumed to provide fewer nutrients than the thin-walled neovasculature at the tumor periphery. Conducting vessels are shown in red, and nonconducting vessels are shown in blue. B. Histopathology from one patient showing tumor front pushing into more normal brain. Note the demarcated margin between tumor and brain parenchyma and the green fluorescent outlines of larger vessels deeper in the tumor. Neovascularization (NV) at the tumor-brain interface can be detected by red fluorescence from the erythrocytes inside the vessels. Bar indicates a scale of 100 micrometers. (From Frieboes et al. [45]; with permission.) 
group quantified the link between cellular and molecular perturbations and the changes in tumor morphology that may correspond to different stages of tumor progression [32].

\section{Mathematical Models of Stem Cells in Cancer}

Tumor-initiating cells (also called cancer stem cells) are cancer cells found within epithelial tumors or hematologic cancers that possess the ability to promote tumorigenesis and metastases. These cells are typically thought of as having unlimited proliferative potential and the ability to give rise to all cell types found in a particular cancer. The recognition of leukemic stem cells prompted further research into other types of cancer, including breast and colorectal cancer. The evolution of colon cancer is based on the behavior of cancer stem cells, including the influence of cancer stem cell activity on crypt dynamics and eventually on colon cancer development [33]. The influence of cancer stem cells on gliomas concluded that the combined effect of progeny proliferation, apoptosis, and motility rates may confer counterintuitive tumor growth rates [34]. Recent modeling work by Galle et al. [35] and Sottoriva et al. [36] have elucidated the impact of stem cells on tumor tissue morphology.

\section{Multiscale Modeling of Tumor Growth}

Tumor growth is a result of events at the intracellular (eg, signaling pathways), intercellular (eg, cell-cell adhesion/ communication), and tissue (eg, mechanical pressure due to host constraints) scales. Mathematical and physical theories provide tools for the analysis of such multiscale phenomena, where the outcome of the interplay between processes at various scales is not trivial. So far, no complete mathematical framework exists to allow the rigorous connection of these multiple scales. A detailed review of the problem of multiscale tumor modeling discussed some recents advances and the difficulties of bridging the scales [13•]. Most current work focuses on linking two scales. For example, one interesting approach consists of using hybrid modeling techniques to couple cellular (individual cells) and tissue (described as a continuum) scales [37], whereas a more common way to couple molecular (signaling pathways) and cellular (individual cells) scales has been derived to model the epithelial-mesenchymal transition for invasive processes [38].

\section{How Good Are the Current Models?}

The current literature demonstrates the extended variety of approaches for modeling cancer, including studies focused on breast cancer (eg, to better understand DCIS morphology and progression [39] or predict tumor size [25]) with more direct surgical applications. The evaluation of the quality of a particular model is a difficult task, a situation further exacerbated when one realizes that different mathematical approaches can reproduce the same experimental results [12]. The choice of an adapted modeling approach has to be dictated by both the scale of interest and the level of detail that is required for a particular problem. This sounds like a simple criterion when one focuses on one specific aspect of cancer; however, modelers face significant difficulties when trying to account for phenomena at various spatio-temporal scales. A good model should offer predictions at multiple levels of detail that can be tested experimentally to ascertain the ability of the model to provide true insights into the biological problem.

Thus far, a common direction in mathematical oncology has been the development of models that focus on potential applications for in vitro experimentation. This approach helps simplify all steps to develop a model (ie, integration of the main mechanisms, calibration, comparison, and validation). However, the approach is limited by the simplification of the in vivo reality, as phenomena observed in vitro under controlled conditions may be oversimplified when compared with the complexity of the in vivo environment, which plays a major role in cancer development. Recent studies have increasingly focused on the integration of the physiologic environment with tumor progression, which is a primary goal of physical oncology. In both in vitro and in vivo studies, a major difficulty lies in the comparison of the theoretical results obtained from modeling with experiments.

Tumor models are based on equations that describe, according to the level of detail and sophistication, tumor growth, nutrient evolution, vessel distribution, extracellular matrix structure and composition, anatomic geometries, and so forth. They require experimental data of various kinds to evaluate the model parameters (eg, proliferation rate, diffusion coefficient of chemical within tissues) for calibration, and to validate the outcome. However, real time acquisition and extraction of most of these data is an extremely difficult task, which is further exacerbated for patient clinical data. Although spatio-temporal measurements are required, the best current scenarios essentially provide static (eg, snapshot of histopathology stainings, MRI scans) and partial information that has to be extrapolated for a relevant comparison. A necessary step forward lies in a mutual understanding of this difficulty by modelers, experimentalists, and clinicians through greater interactions.

Physical oncology strives to uncover and delineate the basic laws of tumor growth from cancer biology and to 
include the relevant mechanisms in the mathematical models. Although current models have proven to be helpful for addressing particular questions in cancer development, such as those previously described in this article, the models to date may not include all the important mechanisms but can help with their identification, as proposed in a recent work by Tektonidis et al. [40] for glioma. However, different regulation mechanisms can equally fit with experimental results [35], which reinforces the need for feedback loops between modelers, experimentalists, and clinicians.

Mathematical and physical modeling has been providing an increasing contribution to the war on cancer. However, achieving the minimal requirements for clinical applications remains a challenge. A key question is how models at disparate scales can be combined and extended to help address practical clinical questions such as where and what to resect, how to optimize radiotherapy, or when and how to administer chemotherapy for maximal clinical effect. In the past, over-optimistic estimations about the future of the field created unrealistic expectations. Researchers had emphasized that mathematical/ physical models could reach such a level of completeness that they would be able to predict the evolution of the disease and conduct the corresponding modeling experiments, supporting the idea that in the course of time, mathematical and physical oncology could evolve as a science analogous to meteorology. Although models have become more sophisticated, the development of clinically relevant models remains a formidable challenge.

\section{Future Directions for Physical Oncology}

There are two key directions in which physical oncology should develop that are relevant to cancer in general but are also of particular interest in addressing specific clinical questions in breast cancer. These directions are the identification of fundamental biophysical laws governing tumor progression and the development of a multiscale modeling framework capable of describing the fundamental laws.

\section{Identification of Fundamental Biophysical Laws}

Mathematical modeling and analysis of cancer growth should be able to incorporate the intrinsic cellular parameters (see Table 2) involved in a growing tumor. In the field of engineering, physics has provided a mathematical framework based upon fundamental rules. For example, these rules involve generic conservation law equations where the description of the material properties is accounted for by constitutive laws. Similarly, we need to
Table 2 Components to consider for clinically relevant modeling of breast cancer

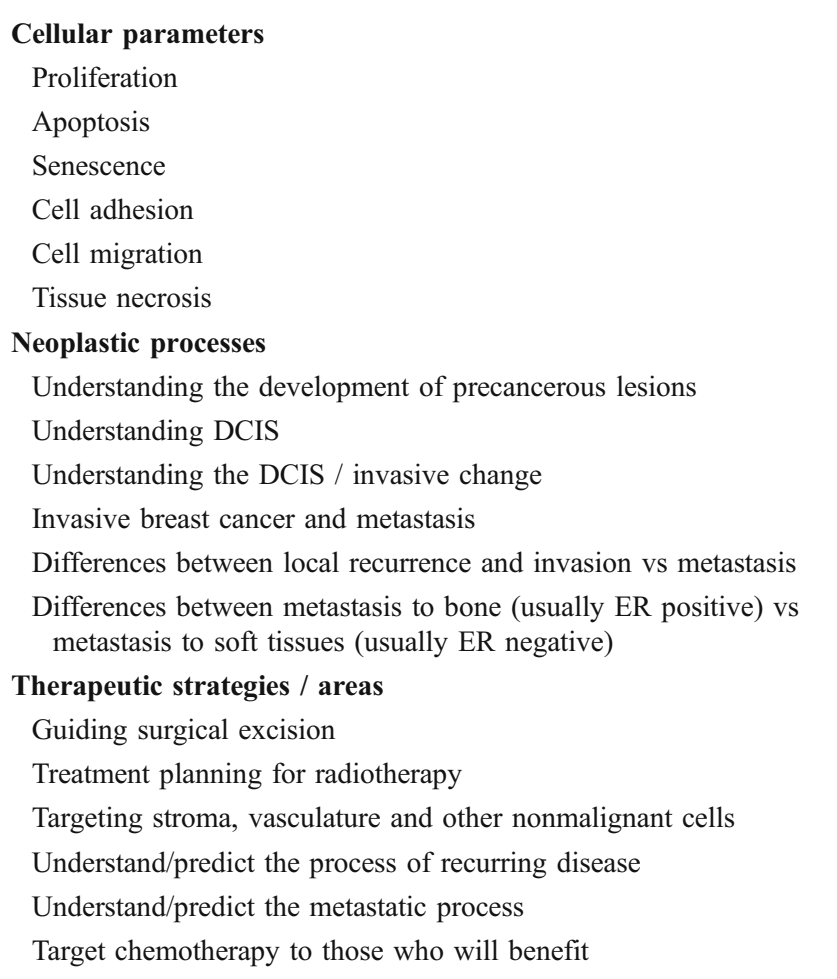

$D C I S$ ductal carcinoma in situ; ER estrogen receptor.

discover the fundamental biophysical and biomechanical mechanisms involved in cancer biology to generate the equivalent of constitutive laws that dictate tumor evolution in the context of tumor growth (eg, by accounting for the extracellular matrix influence [41]). The abundance of biomedical data should support a mature development toward this direction. A perspective of this field in the future, which is both plausible and viable, is the loop-model biological hypothesis, which states that models that can test biological hypotheses can also produce novel theories. Physical oncology may become a platform for the formulation of novel biological hypotheses. The discovery of novel intrinsic biophysical laws would allow for a more complete picture of breast (and other) cancers and support the prediction of tumor growth.

\section{Development of a Physical Multiscale Framework}

The multiscale nature of cancer requires the development of sophisticated mathematical tools [25, 42]. However, many putative multiscale frameworks have simply combined models independently derived at multiple scales and linked them together in a phenomenologic rather than logical manner. There remains a significant need to bridge the interacting processes between the subcellular, cellular, and tissue scales. Therefore, multiscale modeling is one of the 
most important challenges of the next decade for physical oncology, and it should be inspired by existing tools from physics that faced (and sometimes successfully resolved) similar problems $[43 \bullet, 44]$.

\section{Clinical Implications}

The evolution of physical oncology in these directions will help breast cancer researchers address critical therapeutic problems (Table 2). These include identifying biophysical laws that dictate the transition from an in situ neoplastic process, such as DCIS, to an invasive tumor and the importance of tumor-stroma interactions. Most published work has addressed these issues in gliomas (rather than breast cancer), where a similar transition depends on oxygenation levels that favor either invasive or proliferating behaviors $[27,45]$. The study of processes occurring at the interface between the tumor and the host tissue requires a high level of mathematical sophistication. The effect of host tissue stresses (tissue scale) on a single tumor cell (cellular scale), or the impact of single cell mesenchymal motion leading further to collective migration impacting on the tumor morphology, are among the questions that multiscale mathematical tools will be required to address.

The complexity required to describe neoplastic processes (see Table 2) in mathematical terms mainly lies in the involvement of processes at multiple physical and temporal scales. Such a multiscale mathematical framework is required for an understanding of metastasis, from the loss of cell-cell adhesive forces resulting from disrupted molecular pathways and cell intravasation and extravasation events via the vasculature or lymphatics to establishing secondary tumor growth sites.

Therapeutic strategies (see Table 2) may benefit from these developments in physical oncology. Multiscale modeling could assist in guiding the local/regional therapies, by simultaneously predicting the bulk tumor size and location and identifying the most probable locus of strands of invasive cells and enhanced treatment planning of radiotherapy target volumes. Moreover, mathematics could assist in understanding the reasons for radiotherapy or chemotherapy resistance by identifying the responsible biophysical bases.

\section{Conclusions}

In this paper, we have identified fundamental topics and selected key works in mathematical oncology. A critical view of the current state-of-the-art led us to reconsider the future directions of this field as applied to breast cancer. In particular, we believe that future efforts should focus on deciphering the essential biophysical laws that dictate tumor growth. Moreover, a multiscale mathematical theory is required to provide the appropriate framework for developing predictive mathematical models. Finally, we are confident that the development of these directions can help with the comprehension of current challenges and provide solutions for breast cancer.

Acknowledgment The authors thank Y-L Chuang and P. Macklin for constructive discussions and suggestions regarding the manuscript. JL acknowledges partial support from the National Institutes of Health through grant P50GM76516, for a Centre of Excellence in Systems Biology at the University of California, Irvine and grant 1RC2CA148493-01, as well as the National Science Foundation Division of Mathematical Sciences. VC acknowledges support from The Cullen Trust for Health Care, the National Science Foundation, Division of Mathematical Sciences through grant DMS-0818104 and the National Institutes of Health through grants: 1U54CA143837-01, for Multiscale Complex Systems Transdisciplinary Analysis of Response to Therapy (MC-START), and 1U54CA143907-01, for the Center for Transport Oncophysics.

Disclosure No potential conflicts of interest relevant to this article were reported.

Open Access This article is distributed under the terms of the Creative Commons Attribution Noncommercial License which permits any noncommercial use, distribution, and reproduction in any medium, provided the original author(s) and source are credited.

\section{References}

Papers of particular interest, published recently, have been highlighted as:

- Of importance

1. Hanahan D, Weinberg R: The hallmarks of cancer. Cell 2000, 100:57-70.

2. Harris L, Fritsche H, Mennel R, et al.: American Society of Clinical Oncology 2007 update of recommendations for the use of tumor markers in breast cancer. J Clin Oncol 2007, 25:52875312.

3. Al-Hajj M, Wicha MS, Benito-Hernandez A, et al.: Prospective identification of tumorigenic breast cancer cells. Proc Natl Acad Sci 2003, 100:3983-3988.

4. Weigelt B, Bissel MJ: Unraveling the microenvironmental influences on the normal mammary gland and breast cancer. Semin Cancer Biol 2008, 18:311-321.

5. Norton L, Massagué J: Is cancer a disease of self-seeding? Nat Med 2006, 12:875-878.

6. Kim M-Y, Oskarsson T, Acharyya S, et al.: Tumor self-seeding by circulating cancer cells. Cell 2009, 139:1315-1326.

7. Thompson A, Brennan K, Cox A, et al.: Evaluation of the current knowledge limitations in breast cancer research: a gap analysis. Breast Cancer Res 2008 10:R26.

8. Gatenby RA, Gawlinski ET: The glycolytic phenotype in carcinogenesis and tumor invasion: insights through mathematical models. Cancer Res 2003, 63:3847-3854. 
9. Konukoglu E, Clatz O, Bondiau P-Y, et al.: Extrapolating glioma invasion margin in brain magnetic resonance images: suggesting new irradiation margins. Med Image Anal 2010, 14:111-125.

10. Pathmanathan P, Gavaghan DJ, Whiteley JP, et al.: Predicting tumor location by modeling the deformation of the breast. IEEE Trans Biomed Eng 2008, 55:2471-2480.

11. Tracqui P: Biophysical models of tumour growth. Rep Prog Physics 2009, 72:056701.

12. Byrne H: Dissecting cancer through mathematics: from the cell to the animal model. Nat Rev Cancer 2010, 10:221-230.

13. - Lowengrub JS, Frieboes HB, Jin F, et al.: Nonlinear modelling of cancer: bridging the gap between cells and tumours. NonLinearity 2010, 23:R1-R91. This article provides an overview of multiscale cancer modeling. In particular, hybrid modeling is presented, in which the tumor tissue is modeled using both discrete (cell-scale) and continuum (tumor-scale) elements, thus connecting the micrometer scale to the centimeter scale. A review of state-of-the-art of mathematical models of cancer is presented.

14. Gatenby RA, Gawlinski ET, Gmitro AF, et al.: Acid-mediated tumor invasion: a multidisciplinary study. Cancer Res 2006, 66:5216-5223.

15. Frieboes HB, Jin F, Chuang Y-L, et al.: Three dimensional multispecies nonlinear tumor growth II: tumor invasion and angiogenesis. J Theor Biol 2010, 264:1254-1278.

16. Byrne HM, Chaplain MA: Growth of nonnecrotic tumors in the presence and absence of inhibitors. Math Biosci 1995, 130:151181.

17. Adam JA: A simplified mathematical model of tumor growth. Math Biosci 1986, 81:229-244.

18. Zheng X, Wise SM, Cristini V: Nonlinear simulation of tumor necrosis, neo-vascularization and tissue invasion via an adaptive finite-element/level-set method. Bull Math Biol 2005, 67:211259.

19. Cristini V, Frieboes HB, Gatenby R, et al.: Morphologic instability and cancer invasion. Clin Cancer Research 2005, 11:6772-6779.

20. Welter M, Bartha K, Rieger $\mathrm{H}$ : Emergent vascular network inhomogeneities and resulting blood flow patterns in a growing tumor. J Theor Biol 2008, 250:257-280.

21. Sinek JP, Sanga S, Zheng X, et al.: Predicting drug pharmacokinetics and effect in vascularized tumors using computer simulation. J Math Biol 2009, 58:485-510.

22. Frieboes H, Edgerton ME, Fruehauf JP, et al.: Prediction of drug response in breast cancer using integrative experimental/computational modeling. Cancer Research 2009, 69:4484-4492.

23. Swanson KR, Alvord Jr EC, Murray J: A quantitative model for differential motility of gliomas in grey and white matter. Cell Prolif 2000, 33:317-329.

24. Szeto MD, Chakraborty G, Hadley J, et al.: Quantitative metrics of net proliferation and invasion link biological aggressiveness assessed by MRI with hypoxia assessed by FMISO-PET in newly diagnosed glioblastomas. Cancer Res 2009, 69:45024509.

25. Macklin P, Edgerton ME, Cristini V: Agent-based cell modeling: application to breast cancer. In: Cristini $\mathrm{V}$ and Lowengrub J, Multiscale Modeling of Cancer: An Integrated Experimental and Mathematical Modeling Approach. New York: Springer; 2010: 215-244.

26. Nowell PC: The clonal evolution of tumor cell populations. Science 1976, 194:23-28.
27. Hatzikirou H, Basanta D, Simon M, et al.: "Go or grow": the key to the emergence of invasion in tumor progression? Math Med Biol 2010, published online, doi:10.1093/imammb/dqq011.

28. Anderson AR, Weaver AM, Cummings PT, et al.: Tumor morphology and phenotypic evolution driven by selective pressure from the microenvironment. Cell 2006, 127:905-915.

29. - Gatenby RA, Gillies RJ: A microenvironmental model of carcinogenesis. Nat Rev Cancer 2008, 8:56-61. This article provides a mathematical model in which the diverse cancer genotypes and phenotypes can be understood according to their roles as adaptive strategies to overcome specific microenvironmental growth constraints.

30. Bru A, Albertos S, Luis Subiza J, et al.: The universal dynamics of tumor growth. Biophys J 2003, 85:2948-2961.

31. Cristini V, Lowengrub J, Nie Q: Nonlinear simulation of tumor growth. J Math Biol 2003, 46:191-224.

32. Bearer EL, Lowengrub JS, Frieboes HB, et al.: Multiparameter computational modeling of tumor invasion. Cancer Res 2009, 69:4493-4501.

33. van Leeuwen IM, Edwards CM, Ilyas M, et al.: Towards a multiscale model of colorectal cancer. World J Gastroentero 2007, 13:1399-1407.

34. Enderling H, Anderson AR, Chaplain MA, et al.: Paradoxical dependencies of tumor dormancy and progression on basic cell kinetics. Cancer Res 2009, 69:8814-8821.

35. Galle J, Hoffmann M, Aust G: From single cells to tissue architecture - a bottom-up approach to modelling the spatiotemporal organisation of complex multi-cellular systems. J Math Biol 2009, 58:261-283.

36. Sottoriva A, Verhoeff JJC, Borovski T, et al.: Cancer stem cell tumor model reveals invasive morphology and increased phenotypical heterogeneity. Cancer Res 2010, 70:46-56.

37. Kim Y, Stolarska MA, Othmer HG: A hybrid model for tumor spheroid growth in vitro i: theoretical development and early results. Math Mod Meth App Sci 2007, 17:1773-1798.

38. Ramis-Conde I, Drasdo D, Anderson AR, et al.: Modeling the influence of the E-cadherin-beta-catenin pathway in cancer cell invasion: a multiscale approach. Biophys J 2008, 95:155-165.

39. Norton KA, Wininger M, Bhanot $G$, et al.: A $2 D$ mechanistic model of breast ductal carcinoma in situ (DCIS) morphology and progression. J Theor Biol 2010, 263:393-406.

40. Tektonidis M, Hatzikirou $\mathrm{H}$, Chauviere A, et al.: Identification of intrinsic mechanisms for glioma invasion. PLoS Comp Biol 2010 (in press)

41. Preziosi L, Tosin A: Multiphase modelling of tumour growth and extracellular matrix interaction: mathematical tools and applications. J Math Biol 2009, 58:625-656.

42. Multiscale Cancer Modeling. Edited by Deisboeck TS, Stamatakos GS. Boca Raton, FL: Chapman \& Hall/CRC; 2010.

43. - Kevrekidis IG, Samaey G: Equation-free multiscale computation: algorithms and applications. Annu Rev Phys Chem 2009, 60:321-344. This article introduces a multiscale framework, derived in the context of physical sciences, that enables numerical simulations of mathematical models (applicable to cancer modeling) over extended spatio-temporal scales.

44. Weinan E, Engquist B, Xiantao Li, et al.: Heterogeneous multiscale methods: a review. Commun Comput Physics 2007, 2:367-450.

45. Frieboes HB, Lowengrub JS, Wise S, et al.: Computer simulation of glioma growth and morphology. Neuroimage 2007, 37:S59S70. 